\title{
COARSE-GRAINING AS A ROUTE TO MICROSCOPIC PHYSICS: THE RENORMALIZATION GROUP IN QUANTUM FIELD THEORY
}

\author{
BIHUI LI \\ DEPARTMENT OF HISTORY AND PHILOSOPHY OF SCIENCE \\ UNIVERSITY OF PITTSBURGH \\ BBL3@PITT.EDU
}

\begin{abstract}
The renormalization group $(\mathrm{RG})$ has been characterized as merely a coarse-graining procedure that does not illuminate the microscopic content of quantum field theory (QFT), but merely gets us from that content, as given by axiomatic QFT, to macroscopic predictions. I argue that in the constructive field theory tradition, RG techniques do illuminate the microscopic dynamics of a QFT, which are not automatically given by axiomatic QFT. RG techniques in constructive field theory are also rigorous, so one cannot object to their foundational import on grounds of lack of rigor.
\end{abstract}

Date: May 29, 2015. 


\section{INTRODUCTION}

The renormalization group (RG) in quantum field theory (QFT) has received some attention from philosophers for how it relates physics at different scales and how it makes sense of perturbative renormalization (Huggett and Weingard 1995; Bain 2013). However, it has been relatively neglected by philosophers working in the axiomatic QFT tradition, who take axiomatic QFT to be the best vehicle for interpreting QFT. Doreen Fraser (2011) has argued that the RG is merely a way of getting from the microscopic principles of QFT, as provided by axiomatic QFT, to macroscopic experimental predictions. Thus, she argues, RG techniques do not illuminate the theoretical content of QFT, and we should stick to interpreting axiomatic QFT. David Wallace (2011), in contrast, has argued that the RG supports an effective field theory (EFT) interpretation of QFT, in which QFT does not apply to arbitrarily small length scales. Like Wallace, physicists generally regard the RG to be foundationally significant, as recent QFT textbooks indicate (Zee 2010; Duncan 2012).

My main objective is to question Fraser's claims that the RG is only a way to get from the microscopic principles of QFT to macroscopic predictions, and that it has no significance for the theoretical content of QFT. Unlike Wallace, I do this without endorsing an EFT interpretation of QFT. Instead, I elucidate the foundational significance of the RG by describing its role in determining whether various Lagrangians could possibly describe QFTs living on continuous spacetime - that is, whether these Lagrangians are well-defined in the ultraviolet (UV) limit. This problem is an important one in the foundations of QFT and it is the central aim of constructive field theory, which attempts to construct interacting models of QFT satisfying certain axioms. The existence of the UV limit is relevant to whether we should interpret a particular Lagrangian as describing an EFT or as potentially applicable to all length scales, so if the RG helps determine the existence of this limit, then the RG is significant for the interpretation of QFT.

To forestall the objection that RG methods are not rigorous enough for philosophical attention, I look at the RG as used in constructive field theory, a tradition that philosophers take to be rigorous. Many in this tradition use RG methods to determine whether various Lagrangians have a well-defined UV limit. The rigor of these RG methods as compared to the RG methods that physicists typically use lies in the employment of well-controlled approximations rather than ill-controlled approximations.

My plan is as follows. In the next section, I provide more specifics on the various theoretical approaches to QFT and flesh out the claims that I have attributed to Fraser. In Section 3, I sketch the formalism of perturbative QFT, describing the problems that constructive QFT aims to solve. In Section 4, I sketch the "physicists' version" of the RG as a pedagogical attempt to show how the RG can answer the question of whether a UV limit for a given Lagrangian exists. In Section 5, I explain how constructive field theory tries to resolve the problems with perturbative QFT and how it attempts to fill in the mathematical gaps in the physicists' version of the RG. In doing so, I sketch how constructive field theory uses RG methods to try to construct models of QFT that exist in continuous spacetime. I conclude by 
musing on what the argument of this paper implies about the relationship between the various theoretical strands of QFT.

\section{The Debate So FAR}

In the early days of QFT, physicists ran into a host of mathematical pathologies such as divergences in their perturbation expansions. To get around these, they deployed calculational methods such as perturbative renormalization without fully understanding why these methods worked.

Axiomatic QFT grew out of attempts to make the mathematical character of QFT clearer. One variant of axiomatic QFT is algebraic QFT, which I will not discuss here. Instead, I focus on the Wightman axioms or the Osterwalder-Schrader (OS) axioms, which specify the properties that a theory's Wightman functions or Schwinger functions, respectively, must satisfy to define a QFT ${ }^{1}$ However, these properties are insufficient to define a QFT's dynamics. For more dynamical details, we turn to constructive QFT (CQFT). 2 which attempts to construct specific interacting models of QFT that satisfy the OS axioms. Such models, if they exist, automatically satisfy the Wightman axioms, according to the Osterwalder-Schrader reconstruction theorem (Rivasseau 1991). CQFT takes its models of interest to be those characterized by Lagrangians that physicists use. One of the aims of CQFT is to find out if these Lagrangians correspond to non-trivial QFTs in the UV limit.

A QFT that satisfies either set of axioms must have a UV limit: effective field theories violate the axiom of postivity in the OS axioms. A typical approach in CQFT is to start with a lattice QFT or an effective field theory with a momentum cutoff, and then to figure out what happens to the Lagrangian at a fixed momentum scale when the lattice spacing is taken to zero, or when the cutoff is taken to infinity. If the model that results when this limit is taken is trivial (all the coupling constants in the Lagrangian go to zero) or ill-defined (some coupling constant becomes infinite in the limit), then one concludes that there does not exist a non-trivial model of that QFT in continuum spacetime.

The RG was first developed in an unrigorous manner within perturbative QFT. It provides an account of how the dynamics of QFTs change with length or energy scale. These changes are manifested as changes in the value of the coupling parameters in a theory's Lagrangian. Part of the importance of the RG lies in how it explains the empirical success of perturbative renormalization. The RG provides a physical picture of why one has to change the values of coupling parameters in order to avoid divergences. As mentioned earlier, physicists have generally regarded the RG to be foundationally and interpretively significant. In contrast, there is a refrain among philosophers along the lines sketched by Fraser (2011, 131):

$\mathrm{RG}$ methods make a significant contribution to the articulation of the empirical content of QFT and to clarifying the nature of the relationship between the

\footnotetext{
${ }^{1}$ The Schwinger and Wightman functions are important because any observable can be computed from them.

${ }^{2}$ Also known as constructive field theory.
} 
empirical and the theoretical content. However, RG methods do not shed light on the theoretical content of QFT. For this reason, appeal to RG methods does not decide the question of which set of theoretical principles are appropriate for QFT... The reason that constructive field theorists are able to exploit RG methods-even though they reject elements of the theoretical content of LQFT - is that RG methods concern the empirical structure of the theory rather than the theoretical content.

In a similar vein, Kuhlmann, Lyre, and Wayne (2002) characterize the RG as providing "a deductive link between fundamental QFT and experimental predictions". This echoes the thought, latent in Fraser's writings, that there is some "fundamental QFT" given prior to using the RG, presumably by some axiomatic form of QFT, and that all the RG does is link this fundamental theory to experimental predictions. Fraser takes this thought to undercut Wallace's argument that RG methods support a particular interpretation of QFT.

This pattern of reasoning is common in the philosophy of physics: for foundational or interpretive purposes, we should focus on only the "fundamental principles" of a theory, given by its axioms, because these constitute the entire theoretical content of the theory. Methods to extract predictions from these principles add no new theoretical content, only pragmatic filigree.

However, as I shall argue, RG methods do have foundational significance because they are one of the main ways in which CQFT proves the existence or non-existence of models of QFT satisfying the OS axioms. Thus, they bear on the interpretively relevant question of whether certain models of QFT can exist in continuous spacetime. Furthermore, there exist rigorous ways to implement the $\mathrm{RG}$, and these are used in CQFT.

\section{Perturbative Quantum Field Theory}

CQFT arose out of a need to mathematically justify perturbative QFT. In much of QFT, perturbative renormalization is a key technique for deriving finite results for empirically measurable quantities like scattering cross-sections. A first pass at calculating these quantities leads to divergent terms in the relevant perturbation expansions. Perturbative renormalization adjusts the coupling parameters so as to remove these divergent terms. However, this procedure, as presented in introductory QFT textbooks, is carried out on a purely formal basis. While the procedure is justified in one sense by its empirical success, it is not justified by a mathematical understanding of the nature of the perturbative expansion. One of the aims of CQFT is to justify these rules mathematically. In the rest of this section, I offer a brief sketch of perturbative renormalization in a simple case so as to illustrate the room for justification that CQFT tries to provide.

One quantity of central importance in QFT is the partition function, which is defined in terms of the Lagrangian $\mathcal{L}(\phi)$ as follows:

$$
Z=\int \mathcal{D} \phi e^{S[\phi]}
$$


where $S[\phi]=\int \mathcal{L}(\phi) d^{4} x$. Here I have assumed four dimensions for the purpose of the example. The " $\mathcal{D}$ " indicates that this integral is a functional integral, sometimes called a Feynman path integral. Intuitively, the integration ranges over the space of "possible functions" $\phi$, for some value of "possible" 3 Path integrals also feature in expressions for the Green's functions, which are closely related to experimental measurements.

These path integrals can be given a straightforward finite, analytic expression when the action involved is that of a free scalar field with no interactions. In this case, $\mathcal{L}=\frac{1}{2}\left(\left(\partial \phi^{2}\right)^{2}-m^{2} \phi^{2}\right)$. For interacting fields, physicists typically use perturbation theory to evaluate the path integrals. Since the path integral for the free field has a known analytic expression, the perturbations are applied using the free field case as a reference-we consider the interaction as a small perturbation to the free field Lagrangian. The following example illustrates how this is done in a simple case.

Suppose a small interaction $-\frac{\lambda}{4 !} \phi^{4}$ is added to the free field Lagrangian, so that $\mathcal{L}=\frac{1}{2}\left(\left(\partial \phi^{2}\right)^{2}-m^{2} \phi^{2}\right)-\frac{\lambda}{4 !} \phi^{4}$. This is the Lagrangian of the so-called $\phi^{4}$ theory, which describes a self-interacting scalar field. The partition function is

$$
Z=\int \mathcal{D} \phi e^{\int d^{4} x\left(\left(\left(\partial \phi^{2}\right)^{2}-m^{2} \phi^{2}\right)-\frac{\lambda}{4 !} \phi^{4}\right)} .
$$

Assuming $\lambda$ to be small, we then convert the $e^{-\frac{\lambda}{4 !} \phi^{4}}$ factor into a Taylor series in $\lambda$ :

$$
Z=\int \mathcal{D} \phi\left(1-\frac{\lambda}{4 !} \int_{x_{1}} \phi^{2}\left(x_{1}\right) d x_{1}+\frac{1}{2}\left(\frac{\lambda}{4 !}\right)^{2} \int_{x_{1}, x_{2}} \phi^{4}\left(x_{1}\right) \phi^{4}\left(x_{2}\right) d x_{1} d x_{2}+\cdots\right) e^{\int d^{4} x\left(\left(\partial \phi^{2}\right)^{2}-m^{2} \phi^{2}\right)}
$$

where I have included only the first two terms of the Taylor series to illustrate the general rule.

Unlike in the free field case, when evaluating path integrals such as the above, some of the individual terms in the Taylor series are infinite. These divergences make it difficult to directly compute experimentally measurable quantities, such as scattering cross-sections, from the path integral. In many cases, the divergences can be removed by the process of perturbative renormalization. This process starts with regularization, a way of eliminating the influence of high-momenta processes which cause the divergences, and, for some methods of regularization, the addition of counterterms to compensate for regularization. Regularization is typically followed by renormalization, which consists of rewriting the Lagrangian and expressions for quantities like cross-sections in terms of "renormalized" coupling parameters rather than the "bare" parameters that we started with. These methods have proven to be empirically successful for theories like quantum electrodynamics.

Even though perturbative renormalization removes the term-by-term divergences that occur in (2), they leave unresolved other issues. It is suspected that expansions like (2) do not converge and are at best asymptotic. An asymptotic series can be useful if we know which

${ }^{3}$ As we will see later, one of the first tasks of constructive field theory is to give a precise meaning to the measure $\mathcal{D} \phi$. 
function the series is asymptotic to, but perturbative QFT on its own does not provide this information. Part of the CQFT program involves showing that some properties of the non-perturbative solutions to the equations of motion guarantee that certain methods of summing asymptotic perturbative expansions will lead to a unique solution. I will not discuss this part of the CQFT program. The part I will discuss in Section 5.2 involves using the RG to evaluate (1). Here the problem of divergent perturbation series manifests itself as the so-called large field problem, which will also be addressed in Section 5.2.

The other problem with perturbative QFT that CQFT tries to resolve is a proper definition of the measure of (1). Again, we will see in Section 5.2 how this is done in CQFT. For now, I move on to discussing how the RG is important not just as a way to calculate empirical quantities, but also to determine whether a given Lagrangian exists in the UV limit.

\section{The Renormalization Group}

The RG explains perturbative renormalization non-perturbatively. It gives an account of changing coupling parameters that is not based wholly on formal perturbative series and perturbative renormalization. The RG is widely used in the non-rigorous variants of QFT used by physicists and in constructive field theory. For convenience, I follow Wallace (2011) in calling the former "conventional QFT". While the constructive field theory treatment of the RG plugs many mathematical gaps in conventional QFT, the important conceptual insights are already present in the conventional treatment. The conventional understanding of fixed points and RG flows suffices to help us understand how RG techniques give us not just macroscopic information, but also information about the existence of a UV limit. Here, I sketch the RG as typically presented conventionally, explain its significance for foundational questions, and point to the places where a constructive treatment might fill in some gaps. I leave the constructive treatment to Section 5.2 .

The RG is a particularly effective way of computing the partition function (1). Intuitively, the operation of an RG transformation can be thought of as integrating out high-momentum degrees of freedom to obtain an effective action over the remaining low momenta. Formally, this transformation is often written as follows:

$$
\int \mathcal{D} \phi_{L} \int \mathcal{D} \phi_{H} e^{S\left[\phi_{H}, \phi_{L}\right]}=\int \mathcal{D} \phi_{L} e^{S_{\Lambda}\left[\phi_{L}\right]},
$$

where $\phi_{L}$ indicate field configurations whose Fourier transforms have support over momenta less than $\Lambda$, and $\phi_{H}$ indicate field configuations whose Fourier transforms have support over momenta more than $\Lambda . S_{\Lambda}\left[\phi_{L}\right]$ is known as the effective action because it "acts like the full action" $S\left[\phi_{H}, \phi_{L}\right]$ but involves fewer degrees of freedom. It behaves like the full action when we describe our system with a reduced set of variables, that is, with only $\phi_{L}$ instead of $\phi_{L}+\phi_{H}$. This strategy of using effective actions at lower momentum scales to help evaluate the full integral is important in constructive field theory and in less rigorous work within QFT. Roughly speaking, RG methods proceed by doing many such integrations over infinitesimal momentum shells. This is a more effective way of computing the partition function compared to methods that try to integrate over all momenta at once, because many of the expansions that 
we have found to be helpful in evaluating the partition function are effective only at fixed momentum scales.

Denote the transformation (3), taking a more fine-grained action to a more coarse-grained action, by $\mathcal{R}$. The more times we iterate $\mathcal{R}$ on the action $S$, the larger the range of momenta we can integrate over. Each application of $\mathcal{R}$ changes the coupling parameters of terms in the action.$^{4}$ That is, each $\mathcal{R}$ moves $S$ along a trajectory in the space of actions. Sometimes this flow can end up in a fixed point: a point where the transformation maps the action defined by that point in the space of actions to itself. That is, a fixed point is a point $S$ where $\mathcal{R} S=S$.

The existence of a fixed point is important for determining if a given Lagrangian has a UV limit. A continuum theory exists if at an arbitrary fixed momentum scale $\Lambda_{L}$, the effective action $S_{\Lambda_{L}}$ that we calculate using RG transformations converges as the momentum cutoff goes to infinity. That is, suppose we have calculated $S_{\Lambda_{L}}$ by iterating $\mathcal{R}$ many times on an initial action $S_{\Lambda_{U V}}$, where $\Lambda_{U V}$ is a momentum scale higher than $\Lambda_{L}$. We then see what happens to the $S_{\Lambda_{L}}$ that we calculate with iterated $\mathcal{R} \mathrm{s}$ as we increase $\Lambda_{U V}$. The theory associated with $S_{\Lambda_{L}}$ has a UV limit if there is some $S$ for which $\lim _{\Lambda_{U V} \rightarrow \infty} S_{\Lambda_{L}}=S$. That is, it has a UV limit if, as we raise $\Lambda_{U V}$ and have to repeat $\mathcal{R}$ more and more times in order to compute $S_{\Lambda_{L}}$ from increasingly fine-grained actions, we get a stable result for $S_{\Lambda_{L}}$, showing the existence of a fixed point. In this way, the existence of fixed points of a certain sort can help us answer the question about whether various models of QFT can exist in contiuous spacetime.

Importantly, even though it is true that $\mathcal{R}$ only takes us from a more fine-grained, microscopic action to a more coarse-grained, macroscopic action, it is nevertheless the case that we can use $\mathcal{R}$ to determine whether a UV limit exists, by way of the fixed point analysis just described. This reveals the mistake in Fraser's claim that $\mathcal{R}$, as a coarse-graining procedure, can only be a tool to get from the microscopic principles to macroscopic predictions and not a way to illuminate the microscopic content of the theory.

Indeed, in general, the methods used in constructive field theory to determine whether a given Lagrangian exists in the continuum limit all rely on some kind of multiscale analysis for problems with spacetime dimension $D \geq 3$ (Douglas 2011). The phase space analysis of Glimm and Jaffe (1987) is another example of such a multiscale analysis. The importance of the RG and phase space analysis in finding continuum solutions of QFT shows that the fact that a mathematical method implements some kind of scaling does not imply that it is merely a way to get from an already given microscopic physics to a merely "phenomenological" macroscopic physics.

\footnotetext{
${ }^{4}$ This includes the possibility that terms that didn't exist before gain a non-zero coefficient under the transformation.
} 


\section{Constructive Field Theory And the Renormalization Group}

While we saw in the previous section how the RG as expressed in conventional QFT sheds light on the existence of UV limits, constructive field theory distinguishes itself from other means of finding a UV limit by its greater rigor. This rigor consists in:

(1) Making sure that the relevant functional integrals are well-defined;

(2) In computing the functional integrals, making sure that the approximations and expansions used are well-controlled.

I illustrate point 1 in Section 5.1 and point 2 in Section 5.2.

5.1. Functional Integrals in Constructive Field Theory. I now sketch the constructive field theory approach to defining functional integrals. For simplicity, I consider the $\phi^{4}$ theory (with dimension unspecified for now). Constructive field theorists like to operate with Euclidean functional integrals because this allows them to use the theory of Gaussian integrals. Much of the work in defining (1) draws from this probability theory basis. In Euclidean field theory, we can regard the real-valued fields $\phi(x)$ as random variables on the d-dimensional Euclidean space $\mathbb{R}^{d}$. These random variables are associated with a Gaussian measure that is perturbed by an interaction term. The Gaussian measure is associated with the properties of free particles, and the interaction term with interactions between particles.

The Gaussian random field $\phi(x)$ has a mean given by $\int \phi(x) d \mu_{C}(\phi)=0$ and a covariance given by $\int \phi(x) \phi(y) d \mu_{C}(\phi)=\left(-\Delta+m^{2}\right)^{-1}(x, y) \equiv C(x, y)$. We can formally write $C(x, y)=\int_{\mathbb{R}^{d}} \frac{e^{i p(x-y)}}{p^{2}+m^{2}} d p$, which will help us understand ultraviolet regularization later. The Schwinger functions $\langle F(\phi)\rangle$ can be formally written as

$$
\langle F(\phi)\rangle=\frac{1}{Z} \int F(\phi) e^{-V(\phi)} d \mu_{C}(\phi),
$$

where $Z=\int e^{-V(\phi)} d \mu_{C}(\phi)$. In the case of $\phi^{4}$ theory, $V(\phi)=\lambda \int_{\mathbb{R}^{d}} \phi(x)^{4} d x$, where $\lambda$ is a coupling parameter.

The first task of constructive field theory is to modify the above expression for $\langle F(\phi)\rangle$ so that it is well-defined. The measure $d \mu_{C}(\phi)$ is generally not well-defined before the following steps: ultraviolet regularization, infrared regularization, and, in four dimensions, the addition of counterterms ${ }_{5}^{5}$ Ultraviolet regularization is required to ensure that the product of distributions $\phi(x)^{4}$ is well-defined. This is usually done through a momentum cutoff or lattice regularization. For brevity's sake, I outline only the momentum cutoff method. The momentum cutoff is imposed by altering $C(x, y)$ to $C_{\varepsilon}(x, y)=\int_{\mathbb{R}^{d}} \frac{e^{i p(x-y)}}{p^{2}+m^{2}} e^{-\varepsilon|p|^{2}} d p, \varepsilon>0$. Infrared regularization imposes a finite volume $\Lambda$ over which the integral for $V(\phi)$ is to be carried out. So $V(\phi)$ becomes $V_{\Lambda}(\phi)=\lambda \int_{\Lambda} \phi(x)^{4} d x$. Finally, if $d=4$, we have to add a counterterm $\delta V_{\Lambda, \varepsilon}$ to $V_{\Lambda}$, so we have $V_{\Lambda, \varepsilon}=V_{\Lambda}+\delta V_{\Lambda, \varepsilon}$ in the exponent instead ${ }^{6}$

\footnotetext{
${ }^{5}$ In two or three dimensions, the $\phi^{4}$ model is superrenormalizable and no counterterms are needed.

${ }^{6}$ I leave out the details of the form of $\delta V_{\Lambda, \varepsilon}$ for brevity. See Watanabe (2000) for details.
} 
The upshot of all this is that the formal expression (4) is turned into a well-defined expression:

$$
\langle F(\phi)\rangle_{\Lambda, \varepsilon}=\frac{1}{Z_{\Lambda, \varepsilon}} \int F(\phi) e^{-V_{\Lambda, \varepsilon}(\phi)} d \mu_{C_{\varepsilon}}(\phi) .
$$

The task of constructive field theory is to show that this expression has a well-defined limit as $\varepsilon \rightarrow 0$ and $\Lambda \rightarrow \infty$. If this limit exists, then the Lagrangian in question has a UV limit. Multiscale methods allow one to evaluate the integral by decomposing it into momentum scale-indexed parts. This decomposition allows for each scale-indexed part to be evaluated using certain kinds of expansions, without running into problems with the expansions failing when they try to cover too large a momentum range. The RG is one such multiscale method, and we will now see how it works in constructive field theory.

5.2. Applying the Renormalization Group Rigorously. In Section 4 we saw a sketch of the physical ideas behind the RG. Constructive field theorists implement the same ideas using more rigorous mathematics. As with more cavalier implementations of the RG, the existence of a UV limit in constructive field theory is linked to the existence of fixed points of RG transformations. However, many RG methods used in conventional QFT fail to account for the large field problem. Many non-perturbative approaches to the RG make use of non-perturbative approximations that we do not know how to place error bounds on 7

Constructive field theory tries to find the UV limit using approximations that are better controlled than those of conventional QFT. One way to do this is via the exact renormalization group (ERG) ${ }^{8}$ The term "exact" in this context indicates that the RG is implemented non-perturbatively and that the approximations involved are well-controlled. Benfatto, Cassandro, Gallavotti, Nicoló, Olivieri, Presutti, and Scacciatelli (1980), Gawędzki and Kupiainen (1983), Gawȩdzki and Kupiainen (1985), Brydges, Dimock, and Hurd (1995), and Abdesselam (2007) are examples of how the ERG is used in constructive field theory. I now sketch an RG analysis based on integrating out fluctuations over slices of momentum space, showing how one may determine whether a given Lagrangian has a UV limit in this way. ${ }^{9}$

As mentioned in Section 4, the basic idea of the RG is to integrate the functional integral over momentum slices. This avoids the failures of various kinds of expansions when one integrates over a large range of momenta in one step. In the constructive field theory framework this integration can take place by dividing the covariance $C_{\varepsilon}$ into parts that

\footnotetext{
${ }^{7}$ For example, this a defect of the "functional renormalization group" tradition, as Gurau, Rivasseau, and Sfondrini (2014) point out.

${ }^{8} \mathrm{~A}$ note of caution: some who work in the tradition of the functional renormalization group take themselves to be using the "exact" renormalization group, which they take to a term referring to Wilson's non-perturbative understanding of RG flows (Rosten 2012; Bagnuls and Bervillier 2001). However, as explained previously, the lack of precise error bounds on their approximations sets them apart from the constructive field theory tradition.

${ }^{9}$ Besides momentum slice integration, another way of implementing the RG in constructive field theory is the block spin transformation, where one treats the quantum field in a lattice setting.
} 
correspond to momentum slices. Notating $C_{\varepsilon}$ as $D$ for convenience, we have

$$
D=\sum_{k=0}^{N} D_{k},
$$

with independent Gaussian variables $\phi_{k}(x)$ that each have mean 0 and covariance $D_{k}$. Each $\phi_{k}$ corresponds to a fluctuation field of momentum scale $L^{k}$. The slices of measure $D_{k}$ are defined as follows:

$$
\begin{gathered}
D_{k}(x, y)=\int_{\mathbb{R}^{d}} \frac{e^{i p(x-y)}}{p^{2}+m^{2}}\left(\chi\left(L^{-k}\right)-\chi\left(L^{-(k-1)} p\right)\right) d p, \quad k=1,2, \ldots, N, \\
D_{0}(x, y)=\int_{\mathbb{R}^{d}} \frac{e^{i p(x-y)}}{p^{2}+m^{2}} \chi(p) d p,
\end{gathered}
$$

where $\chi(p)=e^{-p^{2}}$ serves as a cutoff function. The $D_{k}$ serve the purpose of scale decomposition because each $D_{k}$ effectively isolates the range of momenta between $L^{k-1}$ and $L^{k}$.

$$
\text { Defining } H(\phi) \equiv H_{N}(\phi)=e^{-V_{\Lambda, \varepsilon}(\phi)}, \phi_{k, 0}=\sum_{j=0}^{k} \phi_{j} \text {, and } D_{k, 0}=\sum_{j=0}^{k} D_{j},
$$
$k=0,1, \ldots, N$, we can define the operation of scaling out higher momenta as follows:

$$
H_{k-1}\left(\phi_{k-1,0}\right)=\int d \mu_{D_{k}}\left(\phi_{k}\right) H_{k}\left(\phi_{k}+\phi_{k-1,0}\right), \quad k=N, N-1, \ldots, 1 .
$$

$H_{k-1}$ is simply the coarse-grained version of $H_{k}$, with the higher momenta integrated out. In an $\mathrm{RG}$ analysis, we would want to iterate this operation of integrating out higher momenta. Before iterating it, however, we rescale the field $\phi_{k-1,0}$ so that it has a wavelength comparable to $\phi_{k}$ 's. The rescaled field is defined as $\tilde{\phi}_{k}(x)=L^{-k(d-2) / 2} \phi_{k}\left(L^{-k} x\right)$. We also rescale the covariance $D_{k}$, the details of which I omit for brevity 10 Then we define the rescaled $H_{k}$ by

$$
\tilde{H}_{k}\left(\tilde{\phi}_{k, 0}\right)=H_{k}\left(\phi_{k, o}\right) \text {. }
$$

This gives us the RG transformation

$$
\tilde{H}_{k-1}\left(\tilde{\phi}_{k-1,0}\right)=\int d \mu_{\tilde{D}_{k}}\left(\tilde{\phi}_{k}\right) \tilde{H}_{k}\left(\tilde{\phi}_{k}(\cdot)+L^{-(d-2) / 2} \tilde{\phi}_{k-1,0}\left(L^{-1} \cdot\right)\right) .
$$

While we have been using the notation $H(\phi)=e^{-V_{\Lambda, \varepsilon}(\phi)}$ for convenience, we can think of the RG transformation as acting on the action $V$. Each transformation consists of the following steps:

(1) Rescaling of the fields;

(2) Integrating over a momentum slice;

(3) Taking the logarithm of $\tilde{H}_{k-1}$ to get the $V$ needed for the next transformation.

\footnotetext{
${ }^{10}$ See $\overline{\text { Watanabe }}(2000)$ for details.
} 
The problem of finding a well-defined Lagrangian in the ultraviolet limit then reduces to seeing if $V$ converges in the limit of infinitely many RG transformations: in the limit of $k \rightarrow \infty$. The convergence of $V$ in this way corresponds to the existence of the fixed point we are looking for, as explained in Section 4

Constructive field theory differs from other ways of implementing the RG in how well it controls the approximations that are involved. For bosonic interactions, the step of taking the logarithm of $\tilde{H}_{k-1}$ is not well-defined for certain values of $\phi$. This is the large field problem. Constructive field theory deals with this by carrying out the transformation only for small fields. The steps of integrating out fluctuations in a momentum slice and taking the logarithm are carried out only for small fields. This means that we can use a cluster expansion for the former step and a Mayer expansion for the latter step. Both these expansions would not be well-controlled in the large field region. There are various methods for controlling the large field region. Because of their complexity, I can only list them here without going into the details: the domination procedure (Feldman, Magnen, Rivasseau, and Sénéor 1987), polymer systems (Pordt 1994), and using the fact that "large fields" occur with a relatively small probability (Balaban, Imbrie, and Jaffe 1984).

\section{CONCLuSiON}

I have argued against a view that the RG in QFT is merely a way to get from the fundamental physics given by axiomatic QFT to macroscopic experimental predictions. Rather, the RG is also an important method in constructive field theory to figure out whether certain Lagrangians have well-defined UV limits that satisfy the axioms that we think a QFT ought to satisfy. Furthermore, the RG as employed in constructive field theory is not of questionable rigor.

The view that I criticise is one in which axiomatic QFT provides the theoretical content of QFT while the RG provides a way to get from this theoretical content to macroscopic empirical predictions. On this view, for interpretive purposes we need only focus on axiomatic QFT. However, constructive field theory provides an important means of access to more of the theoretical content of QFT, with the RG providing a means of access even to the microscopic physics of QFT. This suggests that axiomatic QFT is at best a kind of partial characterization of the theoretical content of QFT. Indeed, mathematical physicists have long acknowledged that constructive QFT provides additional dynamical information that a pure axiomatic approach does not (Wightman 1976, Horuzhy 1990). If so, we should not too hastily dismiss the interpretive significance of computational methods that do not explicitly appear in the axioms of QFT, for these methods may be able to tell us if certain dynamics can occur in continuous spacetime. 


\section{REFERENCES}

Abdesselam, A. (2007). A complete renormalization group trajectory between two fixed points. Communications in Mathematical Physics 276(3), 727-772.

Bagnuls, C. and C. Bervillier (2001). Exact renormalization group equations and the field theoretical approach to critical phenomena. International Journal of Modern Physics A 16(11), 1825-1845.

Bain, J. (2013). Effective field theories. In R. Batterman (Ed.), The Oxford Handbook of Philosophy of Physics, pp. 224-254. New York: Oxford University Press.

Balaban, T., J. Imbrie, and A. Jaffe (1984). Exact renormalization group for gauge theories. In G. 't Hooft, A. Jaffe, H. Lehmann, P. K. Mitter, I. M. Singer, and R. Stora (Eds.), Progress in Gauge Field Theory, pp. 79-103. New York: Plenum Press.

Benfatto, G., M. Cassandro, G. Gallavotti, F. Nicoló, E. Olivieri, E. Presutti, and E. Scacciatelli (1980). Ultraviolet stability in Euclidean scalar field theories. Communications in Mathematical Physics 71(2), 95-130.

Brydges, D., J. Dimock, and T. R. Hurd (1995). The short distance behavior of $\left(\varphi^{4}\right)_{3}$. Communications in Mathematical Physics 172(1), 143-186.

Douglas, M. (2011). Foundations of quantum field theory. In Proceedings of Symposia in Pure Mathematics, Volume 85, pp. 105-124.

Duncan, A. (2012). The Conceptual Framework of Quantum Field Theory. Oxford University Press.

Feldman, J., J. Magnen, V. Rivasseau, and R. Sénéor (1987). Construction and Borel summability of infrared $\phi^{4}$ by a phase space expansion. Communications in Mathematical Physics 109(3), 437-480.

Fraser, D. (2011). How to take particle physics seriously: A further defence of axiomatic quantum field theory. Studies In History and Philosophy of Science Part B: Studies In History and Philosophy of Modern Physics 42(2), 126-135.

Gawȩdzki, K. and A. Kupiainen (1983). Non-Gaussian fixed points of the block spin transformation. Hierarchical model approximation. Communications in Mathematical Physics 89(2), 191-220.

Gawȩdzki, K. and A. Kupiainen (1985). Exact renormalization for the Gross-Neveu model of quantum fields. Physical Review Letters 54, 2191-2194.

Glimm, J. and A. Jaffe (1987). Quantum Physics: A Functional Integral Point of View (2nd ed.). New York: Springer.

Gurau, R., V. Rivasseau, and A. Sfondrini (2014). Renormalization: an advanced overview. Online preprint. http://arxiv.org/abs/1401.5003.pdf.

Horuzhy, S. S. (1990). Introduction to Algebraic Quantum Field Theory. Berlin: Springer.

Huggett, N. and R. Weingard (1995). The renormalisation group and effective field theories. Synthese 102(1), 171-194.

Kuhlmann, M., H. Lyre, and A. Wayne (2002). Introduction. In M. Kuhlmann, H. Lyre, and A. Wayne (Eds.), Ontological Aspects of Quantum Field Theory, pp. 1-29. Singapore: World Scientific. 
Pordt, A. (1994). On renormalization group flows and polymer algebras. In V. Rivasseau (Ed.), Constructive Physics: Results in Field Theory, Statistical Mechanics and Condensed Matter Physics, Volume 446 of Lecture Notes in Physics, pp. 51-81. Berlin: Springer.

Rivasseau, V. (1991). From Perturbative to Constructive Renormalization. Princeton Univ Press.

Rosten, O. J. (2012). Fundamentals of the exact renormalization group. Physics Reports 511(4), 177-272.

Wallace, D. (2011). Taking particle physics seriously: A critique of the algebraic approach to quantum field theory. Studies In History and Philosophy of Science Part B: Studies In History and Philosophy of Modern Physics 42(2), 116-125.

Watanabe, H. (2000). Renormalization group methods in constructive field theories. Int. J. Mod. Phys. B 14(12n13), 1363-1398.

Wightman, A. S. (1976). Hilbert's sixth problem: mathematical treatment of the axioms of physics. In Mathematical Developments Arising from Hilbert Problems, pp. 147-240. Providence, Rhode Island: American Mathematical Society.

Zee, A. (2010). Quantum Field Theory in a Nutshell (2nd ed.). Princeton University Press. 